\title{
El conocimiento tecnológico, pedagógico y disciplinar del profesorado universitario de Educación Física \\ Technological, pedagogical and content knowledge in faculty professors of Physical Education
}

*Laura Ladrón-de-Guevara Moreno, **Julio Cabero-Almenara, ***Bartolomé J. Almagro

*Universidad de Sevilla (España) y Centro de Estudios Universitarios Cardenal Spínola CEU (España), **Universidad de Sevilla (España), ***Universidad de Huelva (España)

\begin{abstract}
Resumen. Debido a la invasión de las tecnologías de la información y comunicación (TIC) en la sociedad actual, en el panorama educativo se presentan grandes oportunidades de integración, pero en ocasiones, el profesorado no aprovecha el potencial que éstas ofrecen. El propósito de este estudio fue analizar el conocimiento tecnológico, pedagógico y disciplinar (TPACK) del profesorado formador de futuros docentes de Educación Física necesarios para la efectiva integración de las TIC en el proceso de enseñanza-aprendizaje, teniendo como referencia uno de los modelos más utilizados, el modelo TPACK. Para ello, se administró un cuestionario a 351 docentes universitarios de los Grados en Ciencias de la Actividad Física y el Deporte y Educación Primaria. Se analizaron las diferencias en el TPACK y en la autoeficacia percibida en el uso del ordenador en cuanto al género, edad, a la existencia de formación específica en TIC, así como las relaciones entre sus variables. Los resultados señalan que la aplicación de la tecnología (TCK+TPK+TPACK) de los docentes encuestados se ha visto influenciada por haber realizado acciones específicas formativas en el terreno de la tecnología. Se encontraron diferencias significativas entre el género y la dimensión tecnológica. Además, el conocimiento tecnológico y la autoeficacia percibida en el uso del ordenador fue significativamente mayor en los docentes de menos de 36 años. Por ello, se destaca la necesidad de formación específica sobre la aplicación de las tecnologías en los docentes universitarios, teniendo en cuenta las peculiaridades de los contenidos del área de Educación Física.
\end{abstract}

Palabras clave. Formación, TPACK, Educación Física, profesores universitarios.

\begin{abstract}
Due to the invasion of information and communication technologies (ICT) in today's society, great opportunities for integration are presented in the educational panorama, but sometimes, teachers don't take advantage of the potential they offer. The aim of this study was to analyze the Technological Pedagogical and Content Knowledge (TPACK) framework of the Faculty professors of pre-service Physical Education teachers necessary for the effective integration of ICT in the teaching-learning process, taking as a reference the TPACK framework, one of the most common models used to measure it. The method included a questionnaire which was submitted to 351 Faculty professors of Bachelor Degree in Physical Education and Sport, and also in Education. TPACK and the computer selfefficacy differences were analyzed in terms of gender, age, the existence of previous ICT training, and the relationship between its variables. The results demonstrated that those professors who had undergone a specific training in ITC showed a better application of the technology (TCK + TPK + TPACK). Significant differences were identified between gender and technological dimension. In addition, the technological knowledge and the computer self-efficacy were significantly higher in teachers under 36 years of age. Given the peculiarities of the Physical Education and Sports subject, this study confirms that it is necessary a specific training for the application of technologies in Faculty professors.
\end{abstract}

Keywords. Training, TPACK, physical education, Faculty professors.

\section{Introducción}

Uno de los problemas existentes en la actualidad consiste en la diferencia entre la fuerte penetración que las Tecnologías de la Información y Comunicación (TIC) están teniendo en las instituciones educativas, tanto en lo que se refiere al aumento de su presencia como a la diversidad de ellas (Johnson, Adams-Becker, Cummins, Estrada, Freeman \& Hall, 2016) y los pocos resultados en la mejora de la calidad educativa que se está teniendo, valga como ejemplo la diversidad de estudios que han señalado la no existencia de relación entre su presencia y la mejora de los resultados académicos (Barrera-Osorio \& Linden, 2009; Unesco, 2016). Esta ineficacia ha sido explicada por diferentes motivos, siendo uno de ellos, la capacitación que los docentes tienen respecto a las TIC (Cabero \& Barroso, 2016; Sola, Nniya, Moreno, \& Romero, 2016; Suárez-Rodríguez, Almerich, Gargallo, \& Aliaga, 2013; Valdivieso \& Gonzáles, 2016), capacitación que se ha centrado más en su componente tecnológico e instru-

Fecha recepción: 27-11-18. Fecha de aceptación: 22-04-19 Laura Ladrón de Guevara Moreno

lguevara@ceuandalucia.com mental que en las propuestas educativas y metodológicas.

En el área de Educación Física se presenta un panorama parecido. La mayoría de los estudios realizados hasta la fecha son estudios descriptivos que se centran en analizar los usos, los niveles de conocimientos y actitudes que poseen los docentes con respecto a la integración de las TIC (Capllonch, 2005; Cuellar \& Delgado, 2010; Ferreres, 2011; Prat, Camerino, \& Coiduras, 2013). Estos usos y niveles de capacitación son altos en los docentes de Educación Física, pero al igual que en el resto de las áreas, se hace necesaria una formación urgente que capacite al profesorado no solo en el uso de las TIC desde un punto de vista técnico, si no conseguir una capacitación pedagógica que asegure dicha integración en el currículo universitario de Educación Física (Cuellar \& Delgado, 2010). Y es en esta perspectiva en la que trata de profundizar el presente estudio y donde entra en acción la propuesta realizada por Mishra y Koehler (2006) y Koehler y Mishra (2008), conocido como modelo (TPACK) (Technological Pedagogical Content Knowledge / Conocimiento Tecnológico, Pedagógico y de Contenido o Disciplinar).

La propuesta asume la idea que, para la capacitación educativa de los docentes y el uso pedagógico de las TIC, no es suficiente con que posean un conocimiento para su 
manejo tecnológico-instrumental, que es lo usualmente realizado, sino que, por el contrario, requieren tres tipos de conocimiento fundamentales para su incorporación a la práctica educativa con garantía: conocimiento pedagógico, tecnológico y de contenidos o disciplinar. Como sugieren Chai, Chin, Koh y Tan (2013), este modelo ha surgido como un marco teórico fundamental que podría ayudar a explicar la complejidad que implica integrar las TIC por parte de los maestros en la enseñanza en el aula.

El modelo establece que los docentes deben presentar un conocimiento tecnológico sobre el funcionamiento tanto general como específico de las TIC y las maneras de utilizarlas, un conocimiento pedagógico sobre la manera de enseñar eficazmente, y un conocimiento sobre el contenido o disciplinar sobre la materia que deben enseñar. Pero lo significativo que propone el modelo y siguiendo la aportación de Cabero-Almenara (2014), es que para que un profesor se encuentre capacitado para la incorporación de las TIC en los escenarios formativos, no es suficiente con la comprensión y percepción de los tres primeros componentes percibidos de forma aislada, sino que también debe percibirlos en interacción con otros conocimientos, dando lugar a los siete constructos que propone el modelo: CK (conocimiento sobre el contenido de la materia o disciplinar), PK (conocimiento pedagógico), TK (conocimiento tecnológico), PCK (conocimiento pedagógico del contenido), TCK (conocimiento de la utilización de las tecnologías), TPK (conocimiento pedagógico tecnológico) y TPACK (conocimiento tecnológico, pedagógico y de contenido). Aspecto que llevará a reclamar que cada uno de los conocimientos requerirá que los docentes tengan diferentes tipos de competencias específicas (Cejas, Navío, \& Barroso, 2016).

El modelo ha adquirido fuerte significación en los últimos años, realizándose aplicaciones tanto en diferentes áreas curriculares (Deng, Sing, So, Qian, \& Chen, 2017; Hu, Walker, \& Hsiao, 2013), como en diferentes niveles de formación de los docentes (Cabero-Almenara, Roig-Vila, \& Mengual-Andrés, 2017; Rienties, et al., 2013), actores participantes en la enseñanza (Sobel \& Grotti, 2013), tecnologías (Anderson \& Barham, 2013; Poitras, Doleck, Huang, Li, \& Lajoie, 2017); o estrategias de enseñanza (Hsu, Liang, \& Su, 2015).

Hasta la fecha, la investigación del modelo TPACK aplicada al área de Educación Física ha sido abordada fundamentalmente en Estados Unidos (e.g. Baert \& Stewart, 2014) y en Turquía (Cengiz, 2015; Semiz \& Ince, 2012). Semiz e Ince (2011) realizaron la primera propuesta de cuestionario adaptando la encuesta TPACK de Schmidt et al. (2009) al área de Educación Física. También se han realizado otros estudios en el área que analizan y examinan las competencias del TPACK pero utilizando otro instrumento diferente de investigación, como la escala TPACK-deep de Yurdakul et al. (2012), también utilizada por Arslan (2015) para evaluar las competencias del TPACK en 1028 profesores de Educación Física en formación de Turquía.

Teniendo como referencia el modelo TPACK, esta investigación tiene como objetivo analizar el conocimiento tecnológico, pedagógico y disciplinar en el profesorado que forma a futuros docentes de Educación Física, concretamente, docentes universitarios de los Grados en Ciencias de la Actividad Física y el Deporte y Educación Primaria con mención en
Educación Física. Además, se pretende conocer si existen diferencias significativas en cuanto a los resultados obtenidos en función del género, la edad, la existencia o no de formación específica y la autoeficacia percibida en el uso del ordenador.

\section{Metodología}

\section{Diseño}

Se trata de un diseño cuantitativo descriptivo de poblaciones mediante encuestas (Montero \& León, 2007) y de tipo transversal, ya que la descripción se hará en un único momento temporal.

\section{Participantes}

El muestreo realizado fue de tipo no probabilístico casual o intencional (Sabariego, 2012), ya que se «utiliza como muestra a individuos a los que se tiene facilidad de acceso dependiendo de distintas circunstancias fortuitas» (Sabariego, 2012, p.148). Los instrumentos de recogida de información se difundieron vía on-line durante el curso académico 20162017. Se tuvieron como referencia los siguientes criterios de inclusión: docentes universitarios de los Grados de Ciencias de la Actividad Física y el Deporte y/o del Grado de Educación Primaria de la mención de Educación Física (formadores de futuros docentes de Educación Física), que impartieran clases en alguna universidad española (tanto pública como privada), y que se encontraran en activo. Tras el análisis, se identificó como población total a 1664 docentes universitarios. El número de instrumentos correctamente cumplimentados fue de 351 (asumiendo un error estimado del 4.65\%), de los cuales 232 eran docentes universitarios del Grado de Ciencias de la Actividad Física y el Deporte, 99 del Grado de Educación Primaria con mención en Educación Física y 20 que impartía clase en ambas titulaciones. Los encuestados pertenecían a 55 universidades de 16 comunidades autónomas diferentes (ver Figura 1). De la muestra total, 244 eran hombres y 107 mujeres de edades comprendidas entre los 24 y 68 años de edad $(M=43.47 ; D T=9.87)$.

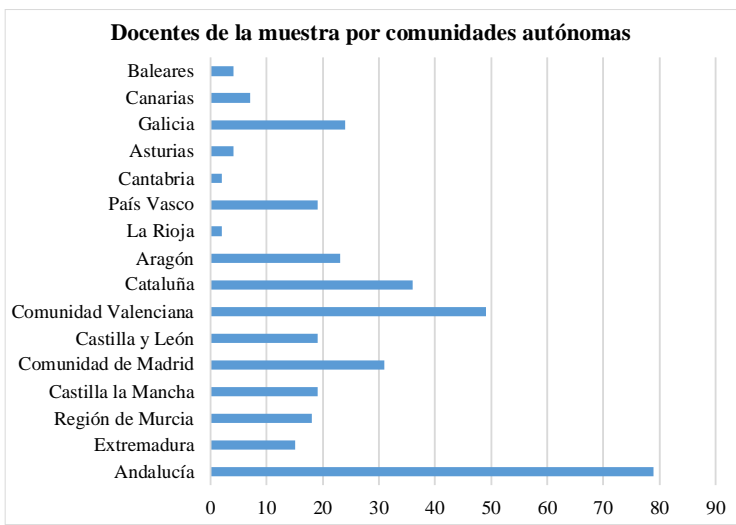

Figura 1. Docentes universitarios encuestados por comunidades autónomas.

\section{Instrumento}

El instrumento de investigación empleado fue la versión española del Cuestionario TPACK para docentes universitarios de Educación Física (Ladrón-de-Guevara, Almagro, \& Cabero-Almenara, 2019) basado en The Survey of Preservice 
Teachers' Knowledge of Teaching and Technology (TPACK survey; Schmidt, el al., 2009), y en la adaptación al área de EF del TPACK survey realizado por Semiz e Ince (2012). El instrumento está formado por 30 ítems con una escala tipo Likert de 1 (muy en desacuerdo) al 5 (muy de acuerdo) y recoge información de cuatro dimensiones: 7 ítems correspondían al conocimiento tecnológico (TK; e.g., «Tengo los conocimientos técnicos que necesito para usar la tecnología»), 3 ítems al conocimiento del contenido (CK; e.g., «Tengo suficientes conocimientos sobre educación física y deportes»), 8 ítems al conocimiento pedagógico y conocimiento pedagógico del contenido (PK+PCK; e.g., «Sé utilizar una amplia variedad de enfoques docentes en mis clases») y 12 ítems al conocimiento tecnológico del contenido, conocimiento tecnológico pedagógico, conocimiento tecnológico pedagógico del contenido (TCK+TPK+TPACK) a la que se denominó aplicación de la tecnología (e.g., «Conozco tecnologías que puedo usar para comprender y elaborar contenidos teóricos sobre educación física y deportes»). El grado de fiabilidad (coeficiente Alfa de Cronbach) obtenida en las cuatro dimensiones fue: .93 para el conocimiento tecnológico (TK); .86 para el conocimiento del contenido (CK), .92 para el conocimiento pedagógico del contenido (PK+PCK) y .95 para el factor de aplicación de la tecnología (TCK+TPK+TPACK).

A dicho cuestionario se le incorporó la Escala de Autoeficacia Percibida en el Uso del Ordenador o Computer Self-Efficacy Scale de Howard (2014). Este instrumento está formado por 12 ítems, a los cuales se responde con escala tipo Likert de 1 (muy en desacuerdo) a 5 (muy de acuerdo). Los ítems de dicha escala medían el nivel de competencia y dominio del manejo del ordenador de manera general (e.g., «Si me esfuerzo lo suficiente siempre puedo resolver problemas informáticos complejos»). El Alfa de Cronbach obtenido en dicha escala fue de .97. Dicho instrumento ya ha sido empleado en otros estudios en España (Fernández-Espínola, Ladrón-de-Guevara, Almagro, \& Rebollo, 2018; García-Pérez, Rebollo-Catalán, \& García-Pérez, 2016; Rebollo-Catalán, VicoBosch, \& García-Pérez, 2015).

El instrumento también midió algunos datos demográficos como universidad en la que imparte clase, la edad, sexo y existencia de formación específica (sobre el uso de las nuevas tecnologías).

\section{Procedimiento}

En primer lugar, se contactó con los secretarios y profesorado de cada uno de los departamentos de las diferentes universidades para pedirles su participación. Se preparó el instrumento de investigación empleado en formato on-line con Google Forms y se envió a los correos electrónicos de toda la muestra. La información necesaria para su correcta cumplimentación quedó recogida tanto en el email como en el propio cuestionario, ya que el investigador no pudo estar presente durante su administración.

\section{Análisis estadístico de los datos}

Con respecto al análisis de los datos, en primer lugar, se realizó un análisis descriptivo de los datos analizando las medias $(M)$ y desviaciones típicas $(D T)$. Para el análisis de las diferencias en cuanto al sexo y a la existencia o no de formación específica en relación a las TIC, se realizó un con- traste de medias no paramétrico a través del estadístico U de Mann-Whitney para grupos independientes (Siegel, 1976), tras aplicar la prueba de Kolmogorov-Smirnov y no cumplir el supuesto de normalidad. Para el análisis de las diferencias en cuanto a la edad, se realizó un contraste de medias no paramétrico para k muestras independientes mediante el estadístico de Kruskall-Wallis. Con el objeto de conocer las diferencias significativas en cuanto al grupo de edad, dependiendo de si se cumplía o no el requisito de homogeneidad de varianzas, se aplicó la prueba post hoc T de Tukey y prueba de Games-Howell. Además, en aquellos contrastes que resultaron significativos, se calcularon los tamaños de los efectos y la potencia de los contrastes. Las asociaciones entre las variables del TPACK se analizaron utilizando el coeficiente de Spearman dada la distribución de la muestra. El nivel de relación se determinó siguiendo las recomendaciones de Cohen (1988). Tales análisis se efectuaron mediante los programas SPSS versión 21 para Windows y G*Power 3.0.

\section{Resultados}

Las puntuaciones medias alcanzadas por los docentes universitarios en cada una de las dimensiones de la versión española del Cuestionario TPACK para docentes de Educación Física fueron las siguientes: Conocimientos tecnológi$\cos \mathrm{TK}(M=3.49 ; D T=.87)$, conocimientos del contenido CK $(M=4.49 ; D T=.65)$, conocimientos pedagógicos $\mathrm{PK}+\mathrm{PCK}$ $(M=4.23 ; D T=.59)$ y conocimientos sobre la aplicación de la tecnología TCK+TPK+TPACK $(M=3.70 ; D T=.73)$. Con respecto a la autoeficacia percibida en el uso del ordenador (AU) fueron $(M=3.32 ; D T=.95)$.

La dimensión con mayor puntuación fue el conocimiento del contenido (CK) lo que demuestra el dominio de la especificidad de la materia al tratarse de docentes universitarios y la de menor puntuación el conocimiento tecnológico (TK) junto con el conocimiento sobre la aplicación de la tecnología (TCK+TPK+TPACK).

A continuación, se analizaron las diferencias significativas en cuanto al género, la edad y la existencia de formación específica en TIC.

\section{Diferencias en cuanto al sexo}

Se analizaron las diferencias significativas en función del sexo de los docentes encuestados para analizar la existencia de diferencias significativas entre hombres y mujeres.

\begin{tabular}{|c|c|c|c|c|c|}
\hline & Sexo & $n$ & Rango promedio & Suma de rangos & $p$ \\
\hline \multirow{3}{*}{ TK } & Masculino & 244 & 186.60 & 45531.00 & \\
\hline & Femenino & 107 & 151.82 & 16245.00 & .003 \\
\hline & Total & 351 & & & \\
\hline \multirow[b]{2}{*}{ CK } & Masculino & 244 & 182.34 & 44492.00 & \\
\hline & $\begin{array}{c}\text { Femenino } \\
\text { Total }\end{array}$ & $\begin{array}{l}107 \\
351\end{array}$ & 161.53 & 17284.00 & .063 \\
\hline \multirow[b]{2}{*}{$\mathrm{PK}+\mathrm{PCK}$} & Masculino & 244 & 177.32 & 43266.00 & \\
\hline & $\begin{array}{c}\text { Femenino } \\
\text { Total }\end{array}$ & $\begin{array}{l}107 \\
351\end{array}$ & 172.99 & 18510.00 & .712 \\
\hline \multirow[b]{2}{*}{ TCK+TPK+TPACK } & Masculino & 244 & 185.14 & 45175.00 & \\
\hline & $\begin{array}{c}\text { Femenino } \\
\text { Total }\end{array}$ & $\begin{array}{l}107 \\
351\end{array}$ & 155.15 & 16601.00 & .011 \\
\hline \multirow[b]{2}{*}{$\mathrm{AU}$} & Masculino & 244 & 185.22 & 45194.50 & \\
\hline & $\begin{array}{c}\text { Femenino } \\
\text { Total }\end{array}$ & $\begin{array}{l}107 \\
351\end{array}$ & 154.97 & 16581.50 & .010 \\
\hline
\end{tabular}

Nota. p: p-value de la prueba U de Mann-Whitney; TK: conocimiento tecnológico; CK: conocimiento del contenido; PK+PCK: conocimiento pedagógico y conocimiento pedagógico del contenido; TCK+TPK+TPACK: aplicación de la tecnología; AU: autoeficacia percibida en el uso del ordenador. 
Para ello, se aplicó la prueba no paramétrica de U de MannWhitney para grupos independientes al no obtener datos que respondieran a la normalidad (Tabla 1 ).

Se puede observar que existen diferencias significativas entre los hombres y las mujeres en conocimientos tecnológicos $(\mathrm{TK})(p=.003)$ en la aplicación de la tecnología $(\mathrm{TCK}+\mathrm{TPK}+\mathrm{TPACK})(p=.011)$ y en la autoeficacia percibida (AU) $(p=.010)$, de modo que los hombres $(M=3.59$ y $D T=$ .86) parecen tener más conocimientos tecnológicos que las mujeres $(M=3.28$ y $D T=.86)$, muestran un mayor uso de las aplicaciones tecnológicas $(M=3.76$ y $D T=.73$, frente a $M=$ 3.56 y $D T=.74$ respectivamente) y se perciben más autoeficaces en el uso del ordenador $(M=3.41$ y $D T=.91$, frente a $M=3.12$ y $D T=.99$ respectivamente). Sin embargo, no existieron diferencias significativas ni en los conocimientos del contenido ni en los pedagógicos.

Así mismo, en cuanto a los tamaños de los efectos y la potencia de los contrastes, los resultados arrojan: para conocimientos tecnológicos, un tamaño del efecto medio-bajo ( $\mathrm{d}$ de Cohen = .36) y una potencia de contraste adecuada (1â = .95), lo cual implica que, si bien existen diferencias significativas, éstas no son muy relevantes; no obstante, la alta potencia permite sostener la corrección de la conclusión estadística. En el caso de la aplicación de la tecnología (d de Cohen $=.27$ y 1 -â $=.95$ ) los resultados son similares al caso anterior. Por último, para la autoeficacia percibida (d de Cohen $=.31$ y 1-â $=.95$ ), las interpretaciones anteriores se siguen manteniendo.

\section{Diferencias en cuanto a la edad}

Para realizar la existencia de diferencias significativas en cuanto a la edad, se dividió la muestra del estudio en tres rangos de edad diferentes: menores de 36 años $(n=98)$, entre 36 y 52 años $(n=188)$ y mayores de 52 años $(n=65)$. Se realizó un contraste de medias no paramétrico para k muestras independientes mediante el estadístico de Kruskall-Wallis (Tabla 2)・•

\begin{tabular}{|c|c|c|c|c|}
\hline 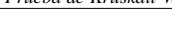 & Edad & $n$ & Rango promedio & $p$ \\
\hline \multirow{4}{*}{ TK } & $<36$ años & 98 & 202.26 & \multirow{4}{*}{.000} \\
\hline & 36-52 años & 188 & 177.07 & \\
\hline & > 52 años & 65 & 133.31 & \\
\hline & Total & 351 & & \\
\hline \multirow{4}{*}{ CK } & $<36$ años & 98 & 193.06 & \multirow{4}{*}{.104} \\
\hline & 36-52 años & 188 & 167.52 & \\
\hline & $>52$ años & 65 & 174.80 & \\
\hline & Total & 351 & & \\
\hline \multirow{4}{*}{$\mathrm{PK}+\mathrm{PCK}$} & $<36$ años & 98 & 168.32 & \multirow{4}{*}{.014} \\
\hline & 36-52 años & 188 & 168.58 & \\
\hline & > 52 años & 65 & 209.05 & \\
\hline & Total & 351 & & \\
\hline \multirow{4}{*}{ TCK+TPK+TPACK } & $<36$ años & 98 & 177.40 & \multirow{4}{*}{.644} \\
\hline & 36-52 años & 188 & 178.92 & \\
\hline & > 52 años & 65 & 165.45 & \\
\hline & Total & 351 & & \\
\hline \multirow{4}{*}{$\mathrm{AU}$} & $<36$ años & 98 & 216.62 & \multirow{4}{*}{.000} \\
\hline & 36-52 años & 188 & 166.92 & \\
\hline & > 52 años & 65 & 141.02 & \\
\hline & Total & 351 & & \\
\hline
\end{tabular}

Además, con el objeto de conocer las diferencias significativas en cuanto a los grupos de edad, dependiendo de si se cumplía o no el requisito de homogeneidad de varianzas, se aplicó la prueba post hoc T de Tukey y prueba de GamesHowell. Se obtuvieron los siguientes datos (Tabla 3):
Tabla 3

Comparaciones múltiples en función de los grupos de edad

\begin{tabular}{|c|c|c|c|c|c|c|c|}
\hline \multirow{2}{*}{$\begin{array}{l}\text { Variable } \\
\text { dependiente }\end{array}$} & \multirow{2}{*}{$\begin{array}{l}\text { (I) Edad } \\
\text { (agrupad) }\end{array}$} & \multirow{2}{*}{$\begin{array}{l}\text { (J) Edad } \\
\text { (agrupado) }\end{array}$} & \multirow{2}{*}{$\begin{array}{c}\text { Diferencia } \\
\text { de medias } \\
\text { (I-J) }\end{array}$} & \multirow{2}{*}{$\begin{array}{l}\text { Error } \\
\text { estándar }\end{array}$} & \multirow{2}{*}{ Sig. } & \multicolumn{2}{|c|}{$\begin{array}{l}95 \% \text { de intervalo } \\
\text { de confianza }\end{array}$} \\
\hline & & & & & & $\begin{array}{l}\text { Límite } \\
\text { inferior }\end{array}$ & $\begin{array}{l}\text { Límite } \\
\text { superior }\end{array}$ \\
\hline \multirow{5}{*}{$\begin{array}{l}\text { TK } \\
\text { (HSD } \\
\text { Tukey) }\end{array}$} & < 36 años & $\begin{array}{c}\text { 36-52 años } \\
>52 \text { años }\end{array}$ & $\begin{array}{l}.20 \\
60^{*}\end{array}$ & .11 & $\begin{array}{l}.138 \\
000\end{array}$ & $\begin{array}{l}-.05 \\
30\end{array}$ & $\begin{array}{l}.45 \\
92\end{array}$ \\
\hline & \multirow{2}{*}{ 36-52 años } & $<36$ años & -.20 & .11 & .138 & -.45 & .05 \\
\hline & & > 52 años & $.39^{*}$ & .12 & .004 & .11 & .68 \\
\hline & \multirow{2}{*}{ > 52 años } & $<36$ años & $-.60^{*}$ & .14 & .000 & -.92 & -.28 \\
\hline & & 36-52 años & $-.39^{*}$ & .12 & .004 & -.68 & -.11 \\
\hline \multirow{6}{*}{$\begin{array}{l}\text { PK+PCK } \\
\text { (HSD } \\
\text { Tukey) }\end{array}$} & \multirow{2}{*}{$<36$ años } & 36-52 años & .01 & .07 & .990 & -.16 & .18 \\
\hline & & > 52 años & -.20 & .09 & .082 & -.42 & .02 \\
\hline & \multirow{2}{*}{ 36-52 años } & $<36$ años & -.01 & .07 & .990 & -.18 & .16 \\
\hline & & > 52 años & $-.21^{*}$ & .08 & .034 & -.41 & -.01 \\
\hline & \multirow{2}{*}{ > 52 años } & $<36$ años & .20 & .09 & .082 & -.02 & .42 \\
\hline & & 36-52 años & $.21^{*}$ & .08 & .034 & .01 & .41 \\
\hline \multirow{6}{*}{$\begin{array}{l}\text { AU } \\
\text { (Games- } \\
\text { Howell) }\end{array}$} & \multirow{2}{*}{ < 36 años } & 36-52 años & $.45^{*}$ & .11 & .000 & .19 & .70 \\
\hline & & > 52 años & $.74^{*}$ & .16 & .000 & .37 & 1.12 \\
\hline & \multirow{2}{*}{ 36-52 años } & $<36$ años & $-.45^{*}$ & .11 & .000 & -.70 & $\begin{array}{l}.19 \\
\end{array}$ \\
\hline & & > 52 años & .30 & .15 & .109 & -.05 & .65 \\
\hline & \multirow{2}{*}{ > 52 años } & $<36$ años & $-.74^{*}$ & .16 & .000 & -1.12 & -.37 \\
\hline & & 36-52 años & -.30 & .15 & .109 & -.65 & .05 \\
\hline
\end{tabular}

En el caso de los conocimientos tecnológicos (TK), aplicando la prueba $\mathrm{T}$ de Tukey, existieron diferencias estadísticamente relevantes $(\mathrm{p}<.001)$ entre los sujetos menores de 36 años $(M=3.71 ; D T=.84)$ y los mayores de $52(M$ $=3.11 ; D T=.84)$ y entre éstos y los sujetos que tienen entre 36 y 52 años (p < .001; $M=3.51 ; D T=.86)$. No así entre los más jóvenes y los de la franja de edad intermedia ( $p=.138)$. Es decir, que el conocimiento tecnológico fue significativamente menor en los sujetos de la muestra con más edad respecto a los otros dos grupos más jóvenes, pero no así entre éstos últimos. Por otro lado, el tamaño de los efectos y la potencia de los contrastes significativos, obtenidos mediante el programa $\mathrm{G} *$ Power, indican que fue grande y adecuada respectivamente en el caso de la primera comparación (d de Cohen = .708 y 1-â = .993), al igual que ocurre en el caso de la segunda ( $d$ de Cohen $=.461$ y 1 -â $=.818$ ), por lo que se constata la relevancia del efecto de la edad sobre el conocimiento tecnológico y la confianza.

Respecto a los conocimientos pedagógicos ( $\mathrm{PK}+\mathrm{PCK})$, existe una única diferencia estadísticamente relevante entre los sujetos acotados entre 36 y 52 años y los mayores de 52 $(p<.05)$, aplicado el estadístico T de Tukey, cuyo tamaño de efecto fue prácticamente mediano (d de Cohen = .461) y adecuada la potencia del contraste (1-â = .891). No se detectaron diferencias importantes entre las demás franjas de edad.

Por último, el grado de autoeficacia percibida (AU) también varía en función de la edad $(p<.001)$. Por ello, para detectar dónde se hallan las verdaderas diferencias, se acudió de nuevo a las comparaciones múltiples a posteriori (post hoc) a través de la prueba de Games-Howell, cuyos resultados indican que los sujetos de menos de 36 años $(M=3.71$; $D T=.82$ ) poseen un mayor nivel de autoeficacia percibida ( $p$ $<.001)$ que los sujetos acotados entre 36 y 52 años $(M=3.51$; $D T=$.86). Así mismo, la significación y el sentido de las diferencias se repiten entre el grupo de menores a 36 años y los mayores de 52 ( $p<.001 ; M=2.86 ; D T=1.03)$, pero no así entre los sujetos acotados entre 36 y 52 años y los mayores de 52 ( $p=$.109). Por tanto, puede afirmarse que existe relación entre la edad y la autoeficacia percibida, especialmente acentuada entre los más jóvenes con respecto a los otros dos grupos de edad. El tamaño del efecto y la potencia de los contrastes significativos obtenidos en este caso ( $G^{*}$ Power) fue mediano e inadecuada en la primera comparación (d de 
Cohen $=.499$ y $1-a ̂=$.695), y alto y adecuada en la segunda $(\mathrm{d}$ de Cohen $=.865$ y 1 -â $=.990)$.

\section{Diferencias en cuanto a la existencia de formación es- pecífica o no}

En cuanto a la existencia de formación específica de los docentes universitarios encuestados, el 46.2\% afirmó haber recibido formación centrada en el uso de las tecnologías. La mayoría de los que realizaron esta afirmación, destacaron haberla recibido como cursos de formación permanente, pero a nivel multidisciplinar. El $14.19 \%$ se consideraron autodidactas y tan solo el 4.32\% de éstos afirmó haber recibido formación específica en tecnologías aplicadas a la actividad física y el deporte.

Se analizaron las diferencias significativas en función de la existencia de formación específica o no en nuevas tecnologías (cursos, talleres, etc.). Al igual que en la variable género, se aplicó la prueba no paramétrica de U de Mann-Whitney para grupos independientes al no obtener datos que respondieran a la normalidad (Tabla 4).

\begin{tabular}{|c|c|c|c|c|}
\hline & $\begin{array}{l}\text { Formación } \\
\text { específica }\end{array}$ & $n$ & Rango promedio & Suma de rangos \\
\hline \multirow{3}{*}{ TK } & No & 188 & 157.75 & 29656.50 \\
\hline & Sí & 162 & 196.10 & 31768.50 \\
\hline & Total & 351 & & \\
\hline \multirow{3}{*}{ CK } & No & 188 & 167.59 & 31507.00 \\
\hline & Sí & 162 & 184.68 & 29918.00 \\
\hline & Total & 351 & & \\
\hline \multirow{3}{*}{$\mathrm{PK}+\mathrm{PCK}$} & No & 188 & 158.52 & 29801.50 \\
\hline & Sí & 162 & 195.21 & 31623.50 \\
\hline & Total & 351 & & \\
\hline \multirow{3}{*}{ TCK+TPK+ТPACK } & No & 188 & 149.17 & 28043.50 \\
\hline & Sí & 162 & 206.06 & 33381.50 \\
\hline & Total & 351 & & \\
\hline \multirow{3}{*}{$\mathrm{AU}$} & No & 188 & 164.45 & 30917.00 \\
\hline & Sí & 162 & 188.32 & 30508.00 \\
\hline & Total & 351 & & \\
\hline
\end{tabular}

Los resultados muestran que, excepto en conocimientos de contenidos (CK), en todos los demás tipos de conocimientos y en la autoeficacia percibida existieron diferencias significativas entre las puntuaciones medias de los sujetos que sí y que no recibieron formación específica. Así, en el caso de conocimientos tecnológicos (TK) $(p<.00)$, obtuvieron mayor puntuación los sujetos con formación específica $(M=3.67 ; D T=.92$ y $M=3.35 ; D T=.76$, respectivamente), al igual que ocurre para la aplicación de la tecnología $(\mathrm{TCK}+\mathrm{TPK}+\mathrm{TPACK})(M=3.93 ; D T=.66$ y $M=3.52 ; D T=.73$, respectivamente). En el caso de los conocimientos pedagógicos (PK+PCK) $(p<.001)$, también los sujetos con formación obtuvieron mayores puntuaciones $(M=4.33$; $D T=.57 \mathrm{y}$ $M=4.14 ; D T=.59$ ). Por último, en la autoeficacia percibida (AU), también los sujetos con formación obtuvieron mayores medias $(p<.05, M=3.45 ; D T=.84$ y $M=3.22 ; D T=1.01$, respectivamente). Es decir, en todas estas significaciones, los sujetos con formación específica obtuvieron mayores conocimientos que los que no la habían recibido.

Para los conocimientos tecnológicos, el tamaño del efecto fue medio-bajo y la potencia del contraste alta (d de Cohen $=.39$ y 1-â $=.95$ ), al igual que ocurrió en conocimientos pedagógicos ( $\mathrm{d}$ de Cohen = .33 y 1-â = .95). Para los conocimientos sobre la aplicación de la tecnología, el tamaño del efecto fue mediano y la potencia alta (d de Cohen = .59 y 1-â =.95). Por último, para la autoeficacia percibida, fue bajo y alta respectivamente $(\mathrm{d}$ de Cohen $=.25$ y 1-â $=.95)$.

\section{Relaciones entre las variables del cuestionario}

A continuación, se aplicó el coeficiente de correlación lineal Rho de Spearman para obtener las correlaciones entre las diferentes dimensiones del cuestionario TPACK y la autoeficacia percibida en el uso del ordenador en docentes de Educación Física (Tabla 5).

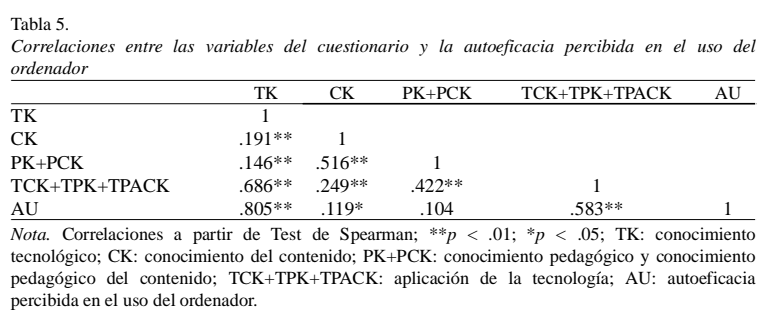

Los resultados arrojan que existe una correlación alta entre PK+PCK y CK $(r=.516)$, entre TCK+TPK+TPACK y TK $(r=.686)$ y entre AU y TK $(r=.805)$ y AU y TCK+TPK+TPACK $(r=.583)$. En cambio, se puede observar una correlación media entre TCK+TPK+TPACK y PK+PCK $(\mathrm{r}=.422)$ y una correlación baja entre CK y TK $(\mathrm{r}=.191)$, entre $\mathrm{PK}+\mathrm{PCK}$ y TK $(\mathrm{r}=.146)$ y entre AU y CK $(\mathrm{r}=.119)$ y AU y $\mathrm{PK}+\mathrm{PCK}(\mathrm{r}=.104)$ de acuerdo a las recomendaciones de Cohen (1988).

\section{Discusión}

Una de las primeras conclusiones se refiere a la alta valoración positiva que los docentes de Educación Física realizan de los diferentes tipos de conocimientos que conforman el modelo TPACK, al igual que los resultados obtenidos por Arslan (2015). Si bien se muestran más competentes en los conocimientos de contenido; la valoración más baja la realizaron en el conocimiento tecnológico. Hecho que tan bien se ve confirmado en que las puntuaciones medias más bajas se obtuvieron en los ítems del instrumento que analizaba la autoeficacia percibida en el uso del ordenador (Howard, 2014). Estos resultados, al igual que los obtenidos por otros estudios en el campo de la Educación Física (Arslan, 2015; Cengiz, 2015; Semiz \& Ince, 2012), permiten proponer la creación de planes específicos para que los docentes adquieran competencias digitales específicas relacionadas con el terreno de la Educación Física, como la propuesta realizada por Juniu (2011) que presenta ejemplos de actividades de aprendizaje basadas en proyectos que se pueden integrar en el currículum de Educación Física, o diversas experiencias basadas en la gamificación en EF como la propuesta por Flores (2019) en la formación inicial del graduado en Ciencias de la Actividad Física y el Deporte.

Se confirma también esta necesidad por el hallazgo obtenido en el trabajo respecto a que la aplicación de la tecnología (TCK+TPK+TPACK) de los docentes encuestados se ha visto influenciado por haber realizado acciones específicas formativas en el terreno de la tecnología; lógicamente tales influencias no se obtuvieron en lo referido al conocimiento de contenido. 
Además, los resultados encontrados apuntan que el conocimiento mostrado por los profesores en lo referido a la dimensión tecnológica (TK) y la percepción de su autoeficacia para el uso de los ordenadores (AU) es superior al de las profesoras. Hallazgo que coincide con lo obtenido por otros autores (Erdogan \& Sahin, 2010; Lin, Tsai, Chai, \& Lee, 2013). Sin embargo, otros estudios realizados recientemente con estudiantes de magisterio Educación Física, muestran que no existen diferencias significativas en el TPACK en general en cuanto al género (Arslan, 2015; Jang \& Tsai, 2012), lo que puede predecir un cambio generacional. En este sentido, este estudio encontró que el conocimiento tecnológico (TK) fue significativamente menor en los sujetos de la muestra con más edad (mayores de 52) respecto a los otros dos grupos más jóvenes. Además, el grado de autoeficacia percibida en el uso del ordenador también varió en función de la edad, los docentes de menos de 36 años poseen un mayor nivel de autoeficacia percibida que los acotados entre 36 y 52 años, o los mayores de 52 . Sin embargo, no se encontraron diferencias significativas entre los docentes de edades comprendidas entre 36 y 52 años y los mayores de 52. Por tanto, puede afirmarse que existe relación entre la edad y la autoeficacia percibida en el uso del ordenador, especialmente acentuada entre los docentes universitarios más jóvenes con respecto a los otros dos grupos de edad. Estos resultados están en la línea con lo encontrado por SuárezRodríguez, Almerich, Díaz-García y Fernández-Piqueras (2012) en profesorado de Educación Primaria y Secundaria, donde también se apreciaba una tendencia general de que a mayor edad menor dominio de los recursos tecnológicos y menor consideración de la integración de los mismos en la práctica diaria. El profesorado más joven (menos de 35 años) es el que presentaba un mayor conocimiento de los distintos recursos tecnológicos.

No se encontraron diferencias significativas en función del género, en lo que respecta al dominio de conocimiento de los contenidos y al conocimiento pedagógico del contenido. Estos resultados coinciden con lo obtenido por Cabero y Barroso (2016) con una amplia muestra de docentes de diferentes niveles educativos y nacionalidades. Parece lógico que no existan diferencias en cuanto al conocimiento del contenido (actividad física, educación física y deporte) y al cómo orientar el aprendizaje del alumnado (conocimiento pedagógico del contenido) en función del género del docente universitario del Grado en Educación Primaria (Mención EF) o del Grado en Ciencias de la Actividad Física y del Deporte.

En resumen, este estudio ha mostrado que los docentes universitarios de Educación Física (de las áreas de Didáctica de la Expresión Corporal y de Educación Física y Deportes) se autoperciben con buenos conocimientos en el contenido y en la pedagogía, pero sus puntuaciones en el conocimiento tecnológico, integración de la tecnología en su enseñanza y la autoeficacia en el uso del ordenador fueron menores (aunque no especialmente bajas: 3.49, 3.70 y 3.32 sobre 5). Los resultados señalan que los conocimientos tecnológicos, pedagógicos, la aplicación de la tecnología (TCK+TPK+TPACK) y la autoeficacia en el uso del ordenador de los docentes encuestados se ha visto influenciada por haber realizado acciones específicas formativas en el terreno de la tecnología. Por otro lado, se encontraron diferencias significativas entre los hombres y las mujeres en conocimientos tecnológicos (TK), en la aplicación de la tecnología (TCK+TPK+TPACK) y en la autoeficacia percibida del uso del ordenador a favor del género masculino. Estos resultados deberán ser estudiados con más detalle en otras investigaciones para entender mejor la causa de estas diferencias. Además, el conocimiento tecnológico y la autoeficacia percibida en el uso del ordenador fue significativamente mayor en los docentes universitarios de menos de 36 años. Por todo ello, es importante la formación específica sobre la aplicación de las tecnologías en los docentes universitarios, teniendo en cuenta las peculiaridades de los contenidos del área de Educación Física.

Esta investigación presenta algunas limitaciones (necesidad de aumentar la muestra del estudio incluyendo otros contextos, uso de un único instrumento autoadministrado, etc.), que tendrá que ser tenidas en cuenta en futuros estudios. De hecho, en base a este trabajo se plantean las siguientes líneas futuras de investigación: replicarlo en otros contextos, como por ejemplo con universidades iberoamericanas, en la línea de lo realizado por Cabero-Almenara (2014); contrastar los resultados con las opiniones que los alumnos tienen del TPACK de sus profesores, y analizar diferencias y similitudes; analizar el TPACK de los docentes mediante otros instrumentos como pueden ser la entrevista en profundidad y la observación no participante, siguiendo las sugerencias establecidas por diferentes autores (Bibi \& Hossain, 2016; Drummond \& Sweeney, 2017; González, 2017) y relacionar las puntuaciones obtenidas en el TPACK con el currículum de estudio ofrecido en las universidades específicas.

\section{Conclusiones}

En definitiva, los docentes universitarios de Educación Física se autoperciben con buenos conocimientos en el contenido y en la pedagogía, pero con algunas carencias en el conocimiento tecnológico y en la integración de la tecnología en su enseñanza. Los docentes que habían recibido una formación específica en el uso de las tecnologías, obtenían mayores puntuaciones medias especialmente en la dimensión aplicación de la tecnología (TCK+TPK+TPACK) en su enseñanza. Los docentes de género masculino presentaron puntuaciones medias mayores en conocimientos tecnológicos (TK), en la aplicación de la tecnología (TCK+TPK+TPACK) y en la autoeficacia percibida del uso del ordenador. Además, el conocimiento tecnológico y la autoeficacia percibida en el uso del ordenador fue significativamente mayor en los docentes universitarios de menos de 36 años.

El estudio realizado contribuye a demostrar la necesidad de formación específica en la integración de las TIC en el profesorado universitario de Educación Física, una formación basada en las necesidades y peculiaridades del área de Educación Física que anteponga la pedagogía a los conocimientos tecnológicos aislados.

\section{Agradecimientos}

La realización de este trabajo ha sido posible gracias a la 
ayuda de investigación pre-doctoral del Centro de Estudios Universitarios Cardenal Spínola CEU. Agradecer también la participación a toda la muestra del estudio.

\section{Referencias}

Anderson, A., \& Barham, N. (2013). Using the TPACK framework to unite disciplines in online learning. Australasian Journal of Educational Technology, 29(4), 549-545. http://dx.doi.org/10.14742/ajet.24.

Arslan, Y. (2015). Determination of technopedagogical content knowledge competencies of preservice Physical Education teachers: A Turkish sample. Journal of Teaching in Physical Education, 34(2), 225-241.

Baert, H., \& Stewart, A. (2014). The effects of role modeling on technology integration within Physical Education Teacher Education. JTRM in Kinesiology. Recuperado de https://goo.gl/NuJa5c

Barrera-Osorio, F., \& Linden, L. (2009). The use and misuse of computers in education: evidence from a randomized experiment in Colombia. Impact Evaluation series n ${ }^{\circ} 29$, Policy Research working paper, 4836. Washington, DC: World Bank.

Bibi, Sh., \& Hossain, Sh. (2016). TPACK in action: A study of a teacher educator's thoughts when planning to use ICT. Australasian Journal of Educational Technology, 33(4), 87.

Cabero-Almenara, J., \& Barroso, J. (2016). ICT teacher training: a view of the TPACK model / Formación del profesorado en TIC: una visión del modelo TPACK. Cultura y Educación, 28(3), 633-663. http://dx.doi.org/ 10.1080/11356405.2016.1203526.

Cabero-Almenara, J. (dir.) (2014). La formación del profesorado en TIC: modelo TPACK. Sevilla: Secretariado de Recursos Audiovisuales y Nuevas Tecnologías de la Universidad de Sevilla. Recuperado de https://idus.us.es/ xmlui/handle/11441/32292

Cabero-Almenara, J., Roig-Vila, R., \& Mengual-Andrés, S. (2017). Conocimientos tecnológicos, pedagógicos y disciplinares de los futuros docentes según el modelo TPACK. Digital Education Review, 32, 73-84. Recuperado de http://revistes.ub.edu/index.php/der/article/view/ 16981/pdf

Capllonch, M. (2005). Las Tecnologías de la Información y la Comunicación en la Educación Física de Primaria: Estudio sobre sus posibilidades educativas. Tesis doctoral. Universitat de Barcelona. Recuperado de https:// bit.ly/2RFJe9v

Cejas, R., Navío, A., \& Barroso, J. (2016). Las competencias del profesorado universitario desde el modelo TPACK (conocimiento tecnológico y pedagógico del contenido). Píxel-Bit. Revista de Medios y Educación, 49, 105119. http://dx.doi.org/10.12795/pixelbit.2016.i49.07.

Cengiz, C. (2015). The development of TPACK, Technology Integrated Self-Efficacy and Instructional Technology Outcome Expectations of pre-service physical education teachers. Asia-Pacific Journal of Teacher Education, 43(5), 411-422.

Chai, C., Chin, C., Koh, J., \& Tan, C. (2013). Exploring Singaporean Chinese language teachers' technological pedagogical content knowledge and its relationship to teachers' pedagogical beliefs. Asian-Pacific Education Researcher, 22(4), 657-666.

Cohen, J. (1988). Statistical power analysis for the behavioural sciences. $2^{\text {nd }}$ ed. NJ: Lawrence Erlmaum Publishers.

Cuellar, M. J., \& Delgado, M.A. (2010). Nuevas tecnologías en la enseñanza universitaria. Un estudio piloto en educación física. Pixel-Bit. Revista de Medios y Educación, 36, 69-79. Recuperado de https://bit.ly/2OR9KOp

Deng, F., Sing, Ch., So, H-J., Qian, Y., \& Chen, L. (2017). Examining the validity of the technological pedagogical content knowledge (TPACK) framework for preservice chemistry teachers. Australasian Journal of Educational Technology, 33(3), 1-14.

Drummond, A., \& Sweeney, T. (2017). Can an objective measure of technological pedagogical content knowledge (TPACK) supplement existing TPACK measures? British Journal of Educational Technology, 48(4), 928-939. https://doi.org/10.1111/bjet.12473

Erdogan, A., \& Sahin, I. (2010). Relationship between math teacher candidates' technological pedagogical and content knowledge (TPACK) and achievement levels. Procedia-Social and Behavioral Sciences, 2(2), 27072711.

Fernández-Espínola, C., Ladrón-de-Guevara, L., Almagro, B. J., \& Rebollo, J.A. (2018). Formación del profesorado de Educación Física en TIC: Modelo TPACK. Escuela Abierta, 21, 65-75. doi:10.29257/EA21.2018.05

Ferreres, C. (2011). La integración de las tecnologías de la información y de la comunicación en el área de la educación física de secundaria: análisis sobre el uso, nivel de conocimientos y actitudes hacia las TIC y de sus posibles aplicaciones educativas. Universitat Rovira i Virgili, Tarragona. Recuperado de http://www.tesisenred. net/handle/10803/52837

Flores, G. (2019). ¿ Jugamos al Súper Mario Bros? Descripción de una experiencia gamificada en la formación del profesorado de Educación Física. Retos, 36, 413-418. Recuperado de https://recyt.fecyt.es/index.php/retos/ article/view/67816/43185

García-Pérez, R., Rebollo-Catalán, M. A., \& García-Pérez, C. (2016). Relación entre las preferencias de formación del profesorado y su competencia digital en las redes sociales. Bordón. Revista de Pedagogía, 68(2), 137-153.

Howard, M. C. (2014). Creation of a Computer Self-Efficacy Measure: Analysis of Internal Consistency, Psychometric Properties, and Validity. Cyberpsychology, Behavior, and Social Networking, 17(10), 677-681. https://doi.org/ 10.1089/cyber.2014.0255

Hsu, C-Y, Liang, J-H., \& Su, Y-C. (2015). The Role of the TPACK in Game-Based Teaching: Does Instructional Sequence Matter? Asia-Pacific Educational Research, 24(3), 463-470. https://doi.org/10.1007/s40299-014-02212.

Hu, H-W., Walker, K., \& Hsiao, W-Y. (2013). Developing elementary pre-service teachers' technological, pedagogical, and content knowledge for learning and teaching division of fractions. International Journal of Technology, Knowledge and Society, 9(2), 185-204. 
Jang, S-J., \& Tsai, M-F. (2012). Exploring the TPACK of Taiwanese elementary mathematics and science teachers with respect to use of interactive whiteboards. Computers \& Education, 59(2), 327-338.

Johnson, L., Adams-Becker, S., Cummins, M., Estrada, V., Freeman, A., \& Hall, C. (2016). NMC Horizon Report: 2016. Higher Education Edition. Austin, Texas: The New Media Consortium.

Juniu, S. (2011). Pedagogical uses of technology in physical education. The Journal of Physical Education, Recreation \& Dance, 82(9), 41-49. https://doi.org/ 10.1080/07303084.2011.10598692

Koehler, J., \& Mishra, P. (2008). Introducing technological pedagogical knowledge. In AACTE (Eds.), The handbook of technological pedagogical content knowledge for educators (pp. 3-28). New York: Routledge for the American Association of Colleges of Teacher Education. Routledge: Taylor \& Francis Group.

Ladrón-de-Guevara, L., Almagro, B. J. y Cabero, J. (2019). Cuestionario TPACK para docentes universitarios de Educación Física. Manuscrito presentado para su publicación.

Lin, T-C., Tsai, C-C., Chai, C. S., \& Lee, M-H. (2013). Identifying science teachers' perceptions of technological pedagogical and content knowledge (TPACK). Journal of Science Education and Technology, 22(3), 325-336.

Mishra, P., \& Koehler, J. (2006). Technological pedagogical content knowledge: A new framework for teacher knowledge. Teachers College Record, 108(6), 1017-1054. doi:10.1111/j.1467-9620.2006.00684.x

Montero, I., \& León, O. G. (2007). A guide for naming research studies in psychology. International Journal of Clinical and Health Psychology, 7(3), 847-862.

Poitras, E., Doleck, T., Huang, L., Li, Sh., \& Lajoie, S. (2017). Advancing teacher technology education using openended learning environments as research and training platforms. Australasian Journal of Educational Technology, 33(3), 32-45.

Prat, Q., Camerino, O., \& Coiduras, J. LL. (2013) Introducción de las TIC en educación física. Estudio descriptivo sobre la situación actual. Apunts. Educación Física y Deportes,113, 37-44. http://dx.doi.org/10.5672/apunts.20140983.es.(2013/3).113.03

Rebollo-Catalán,A., Vico-Bosch,A., \& García-Pérez, R. (2015). El aprendizaje de las mujeres de las redes sociales y su incidencia en la competencia digital. Revista Prisma Social, 15, 122-146. Recuperado de https://goo.gl/VAKsSf

Rienties, B., Brouwer, N., Carbonell, K.B., Townsend, D., Rozendal, A-P., Loo, J., ... Lygo-Baker, S. (2013). Online training of TPACK skills of higher education scholars: A cross-institutional impact study. European Journal of Teacher Education, 36(4), 480-495. https://doi.org/ 10.1080/02619768.2013.801073

Sabariego, M. (2012). El proceso de investigación (parte 2). In R. Bisquerra (coord.), Metodología de la investigación educativa (pp. 127-163). (3a. ed.). Madrid: La Muralla.

Schmidt, D. A., Baran, E., Thompson, A. D., Mishra, P., Koehler, M. J., \& Shin, T. S. (2009). Technological Pedagogical Content Knowledge (TPCK): The development and validation of an assessment instrument for preservice teachers. Journal of Research on Technology in Education, 42(2), 123-149.

Semiz, K., \& Ince, M. L. (2011). Adaptation and validation of teachers' knowledge of teaching and technology, technology integration self-efficacy and outcome expectations questionnaires for preservice PE teachers. In 16th Annual ECSS Congress Book of Abstracts, 49, Liverpool, England.

Semiz, K., \& Ince, M. L. (2012). Pre-service physical education teachers' technological pedagogical content knowledge, technology integration self-efficacy and instructional technology outcome expectations. Australasian Journal of Educational Technology, 28(7), 1248-1265.

Siegel, S. (1976). Estadística no paramétrica aplicada a las ciencias de la conducta. Trillas: México.

Sobel, K., \& Grotti, M. G. (2013). Using the TPACK framework to facilitate decision making on instructional technologies. Journal of Electronic Resources Librarianship, 25(4), 255-262.

Sola, M., Nniya, M., Moreno, A., \& Romero, J. J. (2016). Valoración del profesorado de educación secundaria de la ciudad de Tetuán sobre la formación en TIC desarrollada desde el Ministerio de Educación Nacional. Pixel-Bit. Revista de Medios y Educación, 50, 49-63. http:// dx.doi.org/10.12795/pixelbit.2017.i50.03

Suárez-Rodríguez, J. M., Almerich, G., Díaz-García, I., \& Fernández-Piqueras, R. (2012). Competencias del profesorado en las TIC. Influencia de factores personales y contextuales. Universitas Psychologica, 11(1), 293-309.

Suárez-Rodríguez, J. M., Almerich, G., Gargallo, B., \& Aliaga, F. M. (2013). Las competencias del profesorado en TIC: estructura básica. Educación XX1, 16(1), 39-62. https:// doi.org/10.5944/educxx1.16.1.716

Unesco (2016). Tecnologías digitales al servicio de la calidad educativa. Unesco: Santiago de Chile.

Valdivieso, T. S. \& Gonzáles, M. A. (2016). Competencia Digital Docente: ¿Dónde estamos? Perfil del docente de educación primaria y secundaria. El caso de Ecuador. Pixel-Bit: Revista de Medios y Educación, 49, 57-73. doi:10.12795/pixelbit.2016.i48.09

Yurdakul, I. K., Odabasi, H. F., Kilicer, K, Coklar, A. N., Birinci, G., \& Kurt, A. A. (2012). The development, validity and reliability of TPCK-deep: A technological pedagogical content knowledge scale. Computers \& Education, 58(3), 964-977. doi:10.1016/j.compedu.2011.10.012

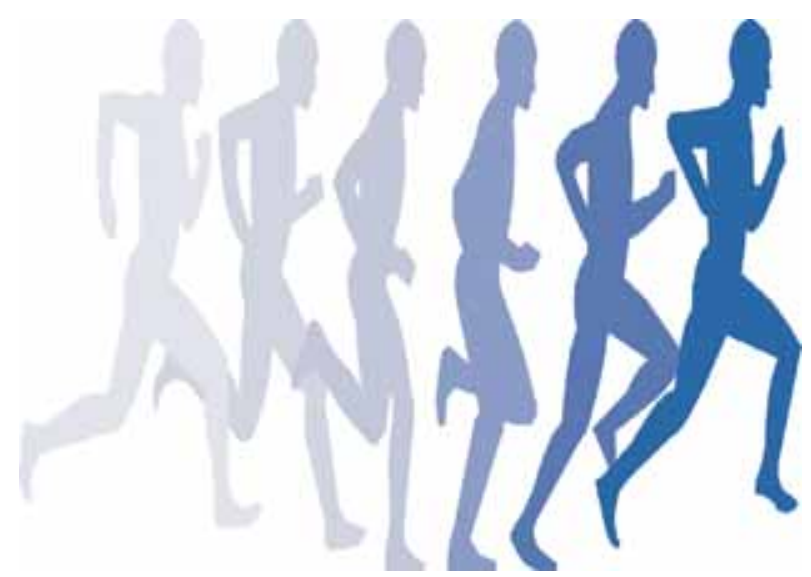

